\title{
Oportunidades alternativas para fincas pequeñas: Producción de durazno y nectarinas versión revisada ${ }^{1}$
}

\author{
Ali Sarkhosh, Mercy Olmstead, Jeff Williamson, Jose Chaparro, Juanita Popenoe; traducido \\ por Eva Pabon ${ }^{2}$
}

El suave clima invernal de Florida y el comienzo de la temprano de primavera ofrecen oportunidades únicas para la producción de duraznos y nectarinas de temporada temprana. Actualmente, Florida produce algunos de los duraznos y nectarinas de florecida temporada para producción comercial en América del Norte (Figura 1). Por ejemplo, los duraznos 'UFSun' y 'UFBest', que están adaptados a la Florida central y sur-central, tienen un período de desarrollo de la fruta (FDP) desde la floración hasta la cosecha de aproximadamente 80 días. Cuando se cultiva en el centro-sur de Florida, la fruta madura a principios de abril y es uno de los primeros duraznos comerciales en madurar en América del Norte. Varios otros cultivares de durazno y nectarinas adaptados al centro-sur y norte de Florida maduran poco después. Durante los últimos 12 años, la Universidad de Florida ha lanzado muchos cultivares nuevos de durazno y nectarina (Tabla 1). Estos nuevos y mejorados cultivares han aumentado el potencial de expansión del aspecto comercial de melocotones y nectarinas en gran parte de la península de Florida y en las regiones de la Costa del Golfo del sureste de los Estados Unidos.

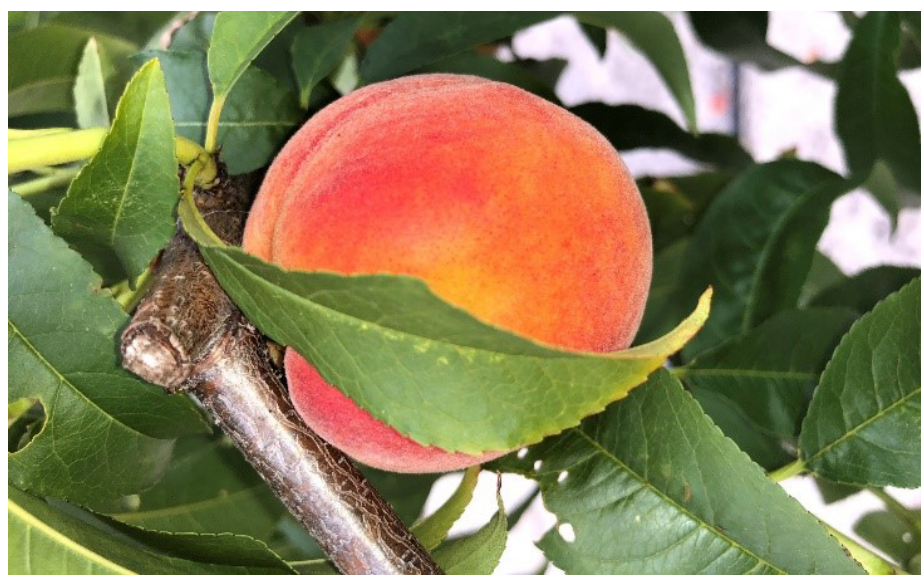

Figura 1. 'UFSun' durazno.

Credits: A. Sarkhosh, UF/IFAS

\section{Requerimiento de frío}

Los duraznos y las nectarinas son árboles frutales de zonas templadas que requieren una exposición mínima de temperaturas frías acumuladas (entre $32^{\circ} \mathrm{F}$ y $45^{\circ} \mathrm{F}$ ) para reanudar su crecimiento normal en la primavera. Esto se conoce como el requerimiento de frío del cultivar (Richardson et al. 1974; Sherman y Rodriguez-Alcazar 1987; Sharpe et al. 1990). El requerimiento de frío generalmente se expresa en horas frío (HF) (toma en cuenta cualquier hora con una temperatura entre $32^{\circ} \mathrm{F}$ y $45^{\circ} \mathrm{F}$ ) en Florida desde octubre hasta la primera semana de enero. Cada cultivar

1. Este documento, RFAC018S, es uno de una serie de publicaciones del Horticultural Sciences, Servicio de Extensión Cooperativa de la Florida, Instituto de Alimentos y Ciencias Agrícolas, Universidad de la Florida. (UF/IFAS). Fecha de primera publicación: octubre 2020. Visite nuestro sitio web EDIS en https://edis.ifas.ufl.edu.

2. Ali Sarkhosh, profesor asistente y especialista en Extensión; Mercy Olmstead, exprofesor asistente; Jeff Williamson, profesor, Departamento de Ciencias Hortícolas; José Chaparro, cultivador de fruta de hueso; Gainesville, FL 32611; Juanita Popenoe, agente de producción de frutas comerciales de varios condados IV, Condado de Lake; Eva Pabon, Servicio de Extensión de UF/IFAS en el condado de Osceola; UF/IFAS Extensión, Gainesville, FL 32611.

The Institute of Food and Agricultural Sciences (IFAS) is an Equal Opportunity Institution authorized to provide research, educational information and other services

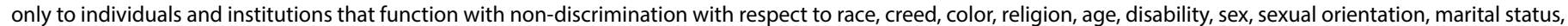

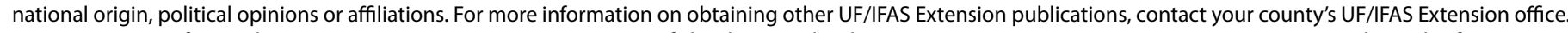
U.S. Department of Agriculture, UF/IFAS Extension Service, University of Florida, IFAS, Florida A \& M University Cooperative Extension Program, and Boards of County Commissioners Cooperating. Nick T. Place, dean for UF/IFAS Extension. 
tiene su propio requisito característico de enfriamiento, que determina parcialmente su adaptabilidad a una determinada región del estado (Tabla 2; Figura 2). La importancia del lugar apropiado y la selección de cultivares debe ser enfatizada. Por ejemplo, "Gulfking" (350 cu) está adaptado al área de Gainesville, pero no recibiría suficiente frío en la mayoría de los inviernos para crecer bien al sur de Ocala. Por el contrario, si el requisito de enfriamiento del cultivar es demasiado bajo para el área donde se planta, existe una mayor probabilidad de que las heladas de fines de invierno maten las flores y los frutos jóvenes. Se pierden más duraznos y nectarinas debido al daño de las heladas en el sureste de los Estados Unidos que por cualquier otra causa.

Tabla 1. Variedades de frutas lanzadas por UF en el programa de mejoramiento nectarinas y duraznos desde 2006.

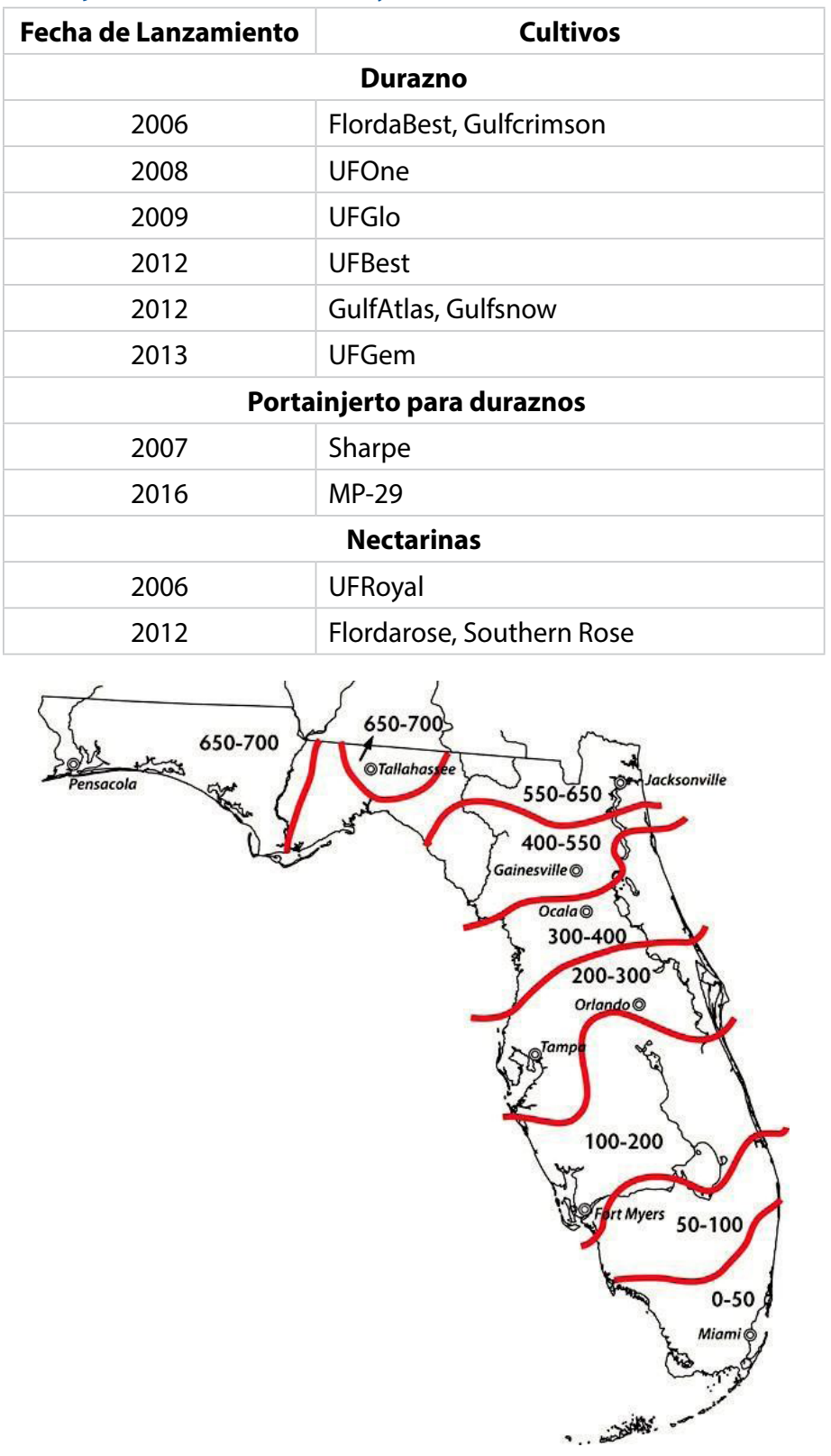

Figura 2. Las unidades de enfriamiento acumuladas (entre $32^{\circ} \mathrm{F}$ y $45^{\circ} \mathrm{F}$ ) hasta el 10 de febrero en el $75 \%$ de los inviernos.

Credits: UF / IFAS
Tabla 2. Requisito de enfriamiento de los cultivares de durazno y nectarina UF.

\begin{tabular}{|c|l|l|}
\hline $\begin{array}{c}\text { Horas frío (HF) } \\
\mathbf{3 2}^{\circ} \mathbf{F}-\mathbf{4 5}^{\circ} \mathbf{F}\end{array}$ & \multicolumn{1}{|c|}{ Cultivares } \\
\hline $500-600$ & \multicolumn{1}{|c|}{ Durazno } & \multicolumn{1}{|c|}{ Nectarina } \\
\hline $400-500$ & $\begin{array}{l}\text { Gulfcrest } \\
\text { Gulfprince, Gulfcrimson, } \\
\text { Gulfsnow,'Flordaking', } \\
\text { 'UFGlo' }\end{array}$ & \\
\hline $300-400$ & $\begin{array}{l}\text { Flordadawn, UF2000, } \\
\text { Flordacrest, Gulfking, } \\
\text { UFSharpe }\end{array}$ & $\begin{array}{l}\text { Sundollar, } \\
\text { 'Suncoast' }\end{array}$ \\
\hline $200-300$ & $\begin{array}{l}\text { UFBeauty, UFGold, } \\
\text { TropicSnow, 'UFOne,' 'UFO', } \\
\text { 'Flordabest' }\end{array}$ & $\begin{array}{l}\text { UFRoyal, Sunraycer, } \\
\text { Sunmist, UFQueen }\end{array}$ \\
\hline $100-200$ & $\begin{array}{l}\text { UfSun, UFBest, UFGem, } \\
\text { Flordaprince, Flordaglo, } \\
\text { TropicBeauty }\end{array}$ \\
\hline & \begin{tabular}{l} 
\\
\hline
\end{tabular} & \\
\hline
\end{tabular}

\section{Situación de Mercadeo}

Los incentivos para el cultivo de duraznos y nectarinos en Florida son los siguientes 1) la producción y mercadeo de duraznos y nectarinos frescos de Florida son antes que de las del centro de Georgia, Carolina del Sur y California lleguen al mercado, y 2) la producción de fruta de calidad cuando casi no hay otra fruta fresca de calidad disponible (Figura 3). Los duraznos y las nectarinas se pueden producir continuamente desde finales de marzo hasta finales de mayo o principios de junio en Florida, dependiendo del clima. A medida que la precipitación aumenta durante los meses de verano, también aumentan los problemas de enfermedades y plagas en duraznos y nectarinos. Cultivares de maduración tardía tienen poco o ningún potencial en Florida debido al aumento de plagas y enfermedades y el aumento de la competencia del mercado.

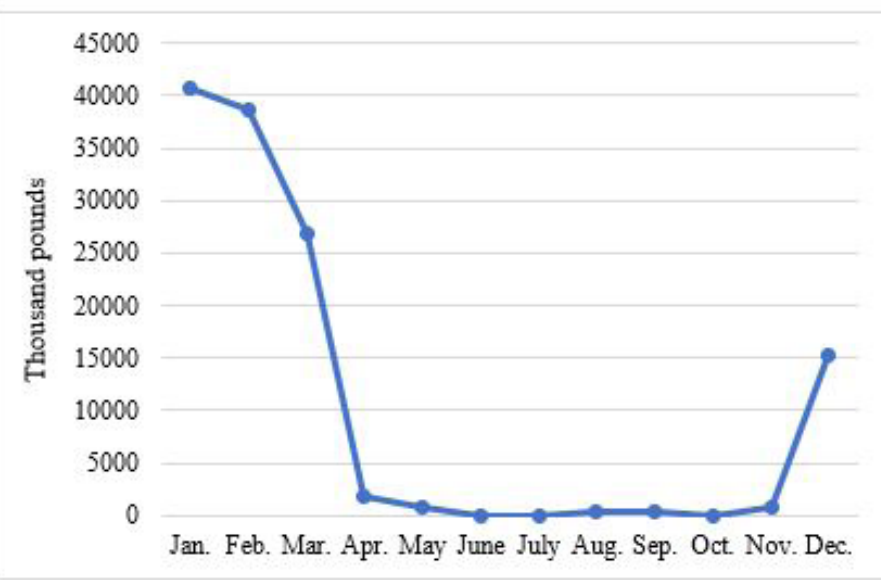

Figura 3. Importaciones mensuales promedio de duraznos y nectarinas (2001-2011).

Credits: Servicio de Investigación Económica, USDA-ARS 
Para mercadear duraznos de alta calidad, es necesario cosechar cada cultivar de tres a cuatro veces con un descanso de dos días para obtener frutos que estén "maduros en los árboles". Cuando se cosechan en esta etapa, el fruto tiene el mejor sabor. Para el envío a larga distancia, la fruta debe ser manejada con más cuidado al momento de, clasificación, enfriamiento y empaque. Esto requiere una inversión considerable en una empacadora, que probablemente no sea económicamente factible para una finca de menos 100-150 acres. Sin embargo, las alternativas de comercialización para los pequeños productores incluyen unirse a una cooperativa de productores, realizar ventas directas a supermercados o mercados de productos, y optar por operaciones de U-pick, mercados de agricultores $\mathrm{u}$ puestos de venta en carretera. Es importante cultivar varios cultivares que maduren sucesivamente para asegurar un flujo constante de fruta madura. Este período de cosecha más largo ayuda con el mercadeo y el uso eficiente de la mano de obra y las instalaciones de empaque.

Las operaciones comerciales de duraznos y nectarinas en el sureste de los Estados Unidos pueden tener costos de producción previos a la cosecha de aproximadamente $\$ 4,000$ por acre (Morgan et al. 2010, M. Olmstead, pers. comm.). Para obtener más información sobre el costo de producción y la rentabilidad de una finca de duraznos, vea la publicación en linea EDIS https://edis.ifas.ufl.edu/fe1016 (Singerman et al. 2017). Estos costos iniciales varían si la tierra ya está comprada y si el riego está disponible. Debido a que los costos de mercadeo de los cultivos comerciales son relativamente altos, las operaciones "cosecha tu propia fruta" tienen el potencial de reducir en gran medida los costos totales de la finca. Los rendimientos estimados (brutos) para los productores de duraznos en Florida son aproximadamente cuatro a cinco veces mayores que los de los productores en los estados vecinos del sudeste (Junta de Estadísticas Agrícolas de los Estados Unidos 2011).

\section{Labor}

La producción de duraznos y nectarinas requiere altos niveles de mano de obra temporera. Los productores comerciales contratan mano de obra para poda durante la época inactiva de los árboles y en el verano, poda selectiva de frutos y cosecha. Las operaciones familiares a menudo proporcionan su propio trabajo. El momento de las operaciones laborales es especialmente crítico; a menudo, un retraso de unos días en la cosecha puede resultar en gran pérdida de ganancias. Actualmente, el mayor costo de producción de duraznos en Florida es la poda selectiva de frutos y la poda manual. Estas prácticas juntas requieren alrededor de 150 horas por acre, dependiendo del tamaño del árbol y del cuaje de frutos, lo que resulta en un costo anual estimado de $\$ 2,000$ / acre.

\section{Pautas de plantación}

Los duraznos y las nectarinas solo se deben plantar en sitios con excelente circulación de aire para reducir el riesgo de daños por heladas en las flores. El aire frío es más denso y se acumula en el punto más bajo del huerto, especialmente en las noches con heladas por radiación. Las heladas por radiación ocurren en noches despejadas con condiciones de viento tranquilo, donde el aire cálido se eleva a medida que el aire frío su acumula cerca del suelo (Figura 4). Una congelación advectiva está dominada por las condiciones de viento y, a menudo, daña severamente las flores y ponteciales frutos. En otra nota, seleccione cultivares con requisitos de enfriamiento un poco menos del enfriamiento promedio recibido de la localización de la finca. Esto ayuda a garantizar un enfriamiento adecuado durante los inviernos inusualmente cálidos; si la finca tiene un excelente flujo de aire, los riesgos de daños a las flores por las heladas primaverales serán mínimos durante los años normales y con mucho frío.

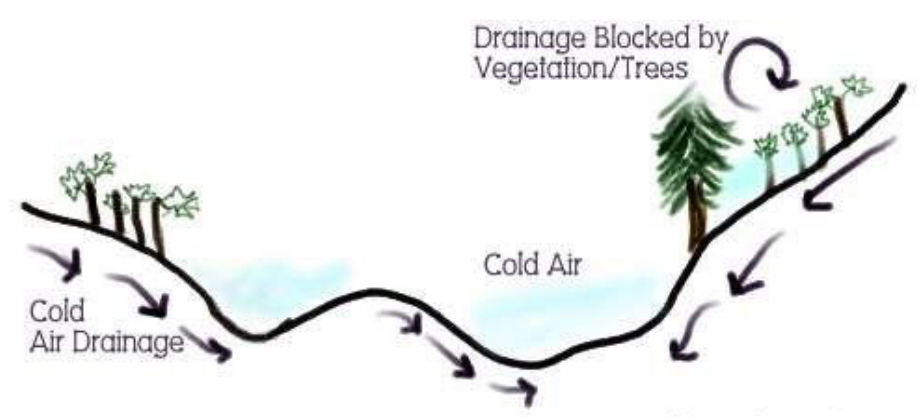

Figura 4. Criterios de selección del sitio para un huerto de frutas de hueso.

Credits: M. Olmstead, UF / IFAS

La selección de la finca (http://agroclimate.org/tools/chillhours-monitoring/) y la elección del cultivar (https://edis. ifas.ufl.edu/mg374) son los dos factores más importantes en el crecimiento exitoso de duraznos y nectarinas. Al seleccionar su lugar de cultivo, evite las áreas bajas caracterizadas por las heladas tardías de primavera. Incluso en el centro de Florida, se pueden tener temperaturas de congelamiento durante febrero y a principios de marzo en lugares fríos; por lo tanto, los sitios con buen fuljo de aire son esenciales para una producción exitosa. Plantar un huerto en el lugar adecuando y la variedad apropiada permitirá a los productores maximizar la eficiencia de su establecimiento con la cantidad mínima de árboles replantados. Los datos meteorológicos históricos y actuales para una áreas específicas de cultivo o finca se pueden encontrar en https://fawn.ifas.ufl.edu/. 
Los duraznos y las nectarinas se pueden cultivar en una amplia variedad de suelos, siempre que tengan buen drenaje en los 4 a 6 pies superiores del suelo. Evite los suelos "rígidos" a menos que se proporcione un excelente sistema de baldosas de drenaje del subsuelo o sistemas de lecho. Se requiere riego para el crecimiento de los árboles y durante todo el período de desarrollo de la fruta para obtener rendimientos y tamaños de fruta aceptables. Un sistema de riego por aspersión diseñado adecuadamente tiene la ventaja adicional de proteger las flores y los frutos jóvenes de las heladas de fines de invierno y primavera. Para sistemas de riego de bajo volumen, se prefieren los microaspersores a los emisores de goteo. Para evitar rociar plántulas / árboles de camiones con agua, se recomiendan microaspersores con un patrón de rociado de 240 a 300 grados.

Los árboles de $2 \frac{1}{2}$ a 4 pies de altura con brotes en junio son buenos para plantar durante la temporada de otoño, sin embargo los árboles de tamaños más grandes son más difíciles de manejar y mas costosos. La distancia de siembra recomendada entre árboles es de $15 \times 20$ pies, o 145 árboles por acre. En suelos más claros, las plantaciones de mayor densidad han sido satisfactorias (Tabla 3). Todos los cultivares de durazno y nectarina de Florida son fructíferos y deben plantarse en bloques sólidos para facilitar la aplicación de pesticidas o fertilizante y la cosecha.

Tabla 3. Ejemplos de distancia de siembras.

\begin{tabular}{|c|c|c|}
\hline $\begin{array}{c}\text { Distancia de siembra } \\
\text { entre árboles (pies) }\end{array}$ & $\begin{array}{c}\text { Distancia de siembra } \\
\text { entre filas (pies) }\end{array}$ & $\begin{array}{c}\text { Total árboles/ } \\
\text { acre }\end{array}$ \\
\hline 15 & 25 & 117 \\
\hline 15 & 20 & 145 \\
\hline 10 & 20 & 218 \\
\hline 10 & 15 & 290 \\
\hline
\end{tabular}

\section{Programa cultural}

El cultivo de duraznos y nectarinas requiere un alto mantenimiento, que incluye poda, la poda selectiva de frutos, cosecha y detección de plagas y enfermedades (Figura 5). Los duraznos y las nectarinas son susceptibles a una serie de plagas, que incluyen enfermedades, insectos y nematodos. Los nematodos agalladores (Meloidogyne incognita y M. javanica) son comunes en los suelos de Florida y pueden afectar negativamente la salud de los árboles de duraznos y nectarinas. Además, el recién identificado nematodo de nudo en la raíz de durazno, $M$. floridensis (Handoo et al. 2004), puede infectar portainjertos de durazno comunes como "Nemaguard" y "Okinawa". Por lo tanto, solo un portainjertos resistente a los nematodos de M. floridensis como el "Flordaguard" debería usarse en Florida (Sherman et al. 1991). Se debe seguir un programa integrado de manejo de plagas para asegurar una buena calidad de la fruta. Aunque algunas enfermedades e insectos pueden ser graves, generalmente se pueden controlar con un programa de manejo de plagas adecuado. Una guía de manejo de enfermedades y plagas está disponible en línea en https:// secure.caes.uga.edu/extension/publications/files/pdf/B\%20 1171_12.PDF. Hay más información disponible sobre duraznos y duraznos en el sitio web de la Universidad de Florida (http://hos.ufl.edu/extension/stonefruit ) y en su oficina local de UF/IFAS Extensión.

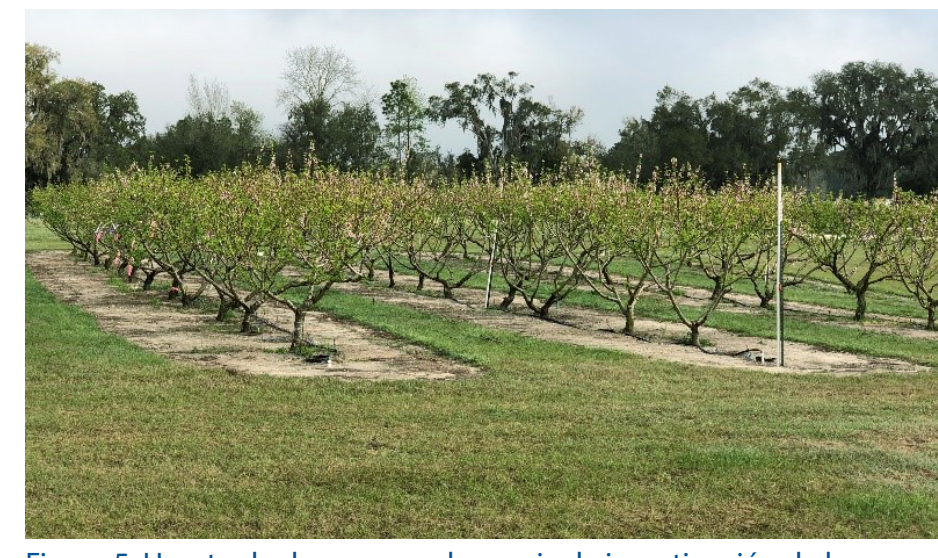

Figura 5. Huerto de duraznos en la granja de investigación de la Universidad de Florida ubicada en Citra.

Credits: A. Sarkhosh, UF/IFAS

\section{Referencias}

Handoo, Z. A., A. P. Nyczepir, D. Esmenjaud, J. G. van der Beek, P. Castagnone-Sereno, L. K. Carta, A. M. Skantar, and J. A. Higgins. 2004. "Morphological, Molecular, and Differential-Host Characterization of Meloidogyne floridensis n. Sp. (Nematoda: Meloidogynidae), a Root-Knot Nematode Parasitizing Peach in Florida." Journal of Nematology 36 (1): 20-35.

Morgan, K., J. Braswell, F. Matta, D. Ingram, and B. Layton. 2010. "Peach - Fruit and Nut Planning Budgets." Starkeville: Mississippi State University.

Richardson, E. A., S. D. Seeley, and D. R. Walker. 1974. "A Model for Estimating the Completion of Rest for 'Redhaven' and 'Elberta' Peach Trees.' HortScience 9 (4): 331-332.

Sharpe, R. H., W. B. Sherman, and J. D. Martsolf. 1990. "Peach Cultivars in Florida and Their Chilling Requirements." Acta Horticulturae 279:191-197.

Sherman, W. B., P. M. Lyrene, and R. H. Sharpe. 1991. “Flordaguard' Peach Rootstock." HortScience 26 (4): 427-428. 
Sherman, W. B., and J. Rodriguez-Alcazar. 1987. "Breeding of Low-Chill Peach and Nectarines for Mild Winters."

HortScience 22:1233-1236.

United States Agricultural Statistics Board. 2011.

"Noncitrus Fruits and Nuts 2010 Summary." http://usda.

mannlib.cornell.edu/MannUsda/viewDocumentInfo.

do?documentID $=1113$ 